\title{
Effectiveness of Respiratory Proprioceptive Neuromuscular Facilitation Techniques on Pulmonary Functions in Patients with Spinal Cord Injury - A Pilot Study
}

\author{
Komal Dattatray Thorat
}

Assistant Professor \& PhD Scholar, Department of NeuroPhysiotherapy, Dr. APJ Abdul Kalam College of Physiotherapy, PIMS, Loni (Bk), 413736, Ahmednagar, Maharashtra, India

\begin{abstract}
Background: Spinal cord injury (SCI) is the injury of the spinal cord from the foramen magnum to the cauda equina which occurs as a result of compulsion, incision or contusion which is a life-threatening condition that carries a high risk of morbidity and mortality. The Incidence of SCI varies from 9.2 to 56.1 per million. In the Indian setup, Approx. 20,000 new cases of SCI are added every year. Spinal cord injury (SCI) patients are at increased risk of chronic respiratory symptoms as Respiratory muscle paralysis both restricts maximum inflation of the lungs and impair the ability to cough. Patients with tetraplegia have decreased chest wall and lung compliance, rib cage stiffness with paradoxical chest wall movements.
\end{abstract}

Material and method: A Pilot study was carried out on 04 Traumatic Spinal cord injury patients. The purpose of the study was to find out effectiveness of respiratory PNF on pulmonary functions (FVC \& $\mathrm{FEV}_{1}$ ) \& Chest Expansion in patients with spinal cord injury. The pulmonary Functions (FVC \& FEV ${ }_{1}$ ) and chest expansion was measured by using Spirometer (PFT machine) and inch tape. The data was analyzed using standard statistical software.

Result: In this study After 4 weeks of Intervention there was Significant Improvement in Pulmonary Functions (Pre Mean of FEV1 \& FVC $0.99 \mathrm{~L} \&$ 1.03L and Post Mean of FEV1 \& FVC was $1.25 \mathrm{~L} \& 1.35 \mathrm{~L}$ respectively) and Chest Expansion (Pre Mean was $1.15 \mathrm{~cm}$ and Post Mean was $1.62 \mathrm{~cm}$ at Xiphoid Process Level).
Conclusion: This Study concluded that Respiratory PNF increases Pulmonary Functions and chest expansion in Patients with Spinal Cord Injury after 4 weeks of Intervention.

Keywords: Spinal Cord Injury, Forced Expiratory Volume (FEV1), Forced vital Capacity, Peak expiratory flow rate, Respiratory Proprioceptive Neuromuscular Facilitation Technique.

\section{INTRODUCTION}

Spinal cord injury (SCI) is the injury of the spinal cord from the foramen magnum to the cauda equina which occurs as a result of compulsion, incision or contusion. Spinal cord injury (SCI) is a life threatening condition that carries a high risk of morbidity and mortality The most common causes of SCI in the world are traffic accidents, gunshot injuries, knife injuries, falls and sports injuries. ${ }^{[1]}$

Various epidemiological studies have been carried out in different parts of the world. The incidence of SCI varies from 9.2 to 56.1 per million, which is influenced not only by research methodology but also by social, economic, geographical, demographic and political characteristics of the region. In the Indian setup, as in most developing countries, very little is known about the exact incidence of spinal cord injuries (SCI). Approximate 20,000 new cases of SCI are added every year. ${ }^{[2,3,4]}$ 

pulmonary functions in patients with spinal cord injury - a pilot study.

People With Spinal cord injury (SCI) are at increased risk of chronic respiratory symptoms, added disability, and early death from respiratory complications where respiratory function following a SCI is primarily determined by the extent and level of neurological injury, due to the partial or complete paralysis of respiratory muscles innervated below the neurological level of injury. There are 4 main muscle groups involved in respiration: Diaphragm (Phrenic Nerve Cervical 3-5), Accessory muscles of neck and shoulder girdle (C1C8), Intercostals (T1-T12) and Abdominals (T6 - T12). ${ }^{[5,6]}$

Respiratory muscle paralysis both restricts maximum inflation of the lungs and impairs the ability to cough, leading to increased risk of atelectasis and retained mucus secretions. Obstructive pulmonary dysfunction is also of concern, not only because airways may collapse or be clogged by mucus, but also because they may be especially susceptible to constriction. Injury to the cervical and upper thoracic spinal cord disrupts function of inspiratory and expiratory muscles, as reflected by reduction in spirometric and lung volume parameters and static mouth pressures. In association, subjects with tetraplegia have decreased chest wall and lung compliance, increased abdominal wall compliance, and rib cage stiffness with paradoxical chest wall movements, all of which contribute to an increase in the work of breathing. Expiratory muscle function is more compromised than inspiratory muscle function among subjects with tetraplegia and high paraplegia, which can result in ineffective cough and propensity to mucus retention and atelectasis. Subjects with tetraplegia also demonstrate heightened vagal activity with reduction in baseline airway caliber, findings attributed to loss of sympathetic innervation to the lungs. ${ }^{[7,8]}$

There is a high incidence of respiratory complications following SCI, which are one of the leading causes of hospital readmission and mortality. Common respiratory complications include atelectasis (segmental lung collapse), pneumonia and respiratory failure. Measures of respiratory function do not return to pre-injury levels, and there is a degree of ongoing impairment of respiratory function that is life-long. Vital Capacity assessment is a reliable measure to monitor respiratory function and stability, both in the acute and long-term stages following SCI. Respiratory muscle strength and endurance can be improved by various breathing exercises such as respiratory muscle training, pursed lip breathing and diaphragmatic breathing. ${ }^{[8,9]}$

Where over all Physiotherapy rehabilitation in Spinal cord injury consist of gait training, balance training, coordination exercises, stretching and strengthening exercises, spasticity management, bowel and bladder management and respiratory management. Respiratory physiotherapy management consist of manually cough assisted techniques, glossopharyngeal breathing, diaphragmatic breathing, balloon blowing exercises, inspiratory muscle training, chest mobility exercises, Respiratory proprioceptive neuromuscular facilitation technique. $^{[10,11]}$

Proprioceptive neuromuscular facilitation techniques consist of intercostal stretch, vertebral pressure to thoracic spine, anterior stretch and posterior basal lift and abdominal co-contraction. This Proprioceptive and tactile stimuli produce remarkably consistent reflexive responses in ventilatory muscles. ${ }^{[12]}$

The Proprioceptive neuromuscular facilitation technique is defined as a positive functional approach to help patients achieve their highest level of function. Breathing problems can be a result from both disturbed inspiration and expiration phases. To improve breathing, the related structures involving diaphragmatic, sternal and coastal areas should be treated.

The physiological mechanism that enables the initiation of inspiration is thought to be the stretch reflex. The stretch reflex resists the change in muscle length by 
contracting the stretched muscle fiber via its muscle spindle (proprioceptor). Appropriate resistance during applying for one of the PNF techniques strengthens the muscles and guides the chest motion. ${ }^{[13]}$

\section{METHODOLOGY}

A pilot study was carried out in 4 spinal cord injury patients, The patients were selected by simple random sampling. The inclusion criteria was age group of 20 to 60 years, Both male and female patients with Cervical \& Thoracic traumatic spinal cord injury. The Exclusion criteria were Chest Trauma \& Rib fracture, NonTraumatic spinal cord injury, patient who needs ventilatory assistance, Hemodynamic ally unstable patients, other neurological condition, K/C/O Respiratory Disease and Associated Neurological condition. Informed consent was taken. The assessment of pulmonary function (FEV1/FVC, PEFR) and chest expansion was done for the spinal cord injury patients.

\section{PROCEDURE:}

Patients were divided into two groups Group A received Respiratory PNF and conventional PT whereas Group B received Conventional $\mathrm{PT}$

\section{Group A Respiratory PNF Received following Protocol:}

(Intercostal stretch, Posterior basal lift, Abdominal co-contraction)

10-12 repetitions of each tech in 1 set (1 min rest after 5-6 repetitions)

5 sets in one Session

1 sessions in a day

6 days/Week for 4 weeks

\section{Conventional Physiotherapy:}

Strengthening exercises, balance training, gait training, Glossopharyngeal breathing Diaphragmatic breathing, Balloon blowing exercises, Inspiratory muscle training Chest mobility exercise

\section{RESULT}

The data was collected and analysis was done by taking the Mean and Standard Deviation (S.D) of FEV1/FVC, PEFR and chest expansion readings. The study was done in Smt. Sindhutai E. Vikhe Patil Spinal Cord Injury Rehab Centre at Pravara Rural Hospital, Loni where 04 patients were included in the study. The pulmonary function parameters were assessed by Spirometer test (PFT). In this study After 4 weeks of Intervention there was Significant Improvement in Pulmonary Functions (Pre Mean of FEV1 \& FVC 0.99 L \& 1.03L and Post Mean of FEV1 \& FVC was 1.25 L \& 1.35L respectively) and Chest Expansion (Pre Mean was $1.15 \mathrm{~cm}$ and Post Mean was $1.62 \mathrm{~cm}$ at Xiphoid Process Level)

\section{Pulmonary functions (FEV1 \& FVC)}

- Pre Intervention the Mean of FEV1 was $0.99 \mathrm{~L}$ And pre mean of FVC was 1.03L

- Post Intervention the Mean of FEV1 was 1.25 L \& FVC was 1.35L respectively

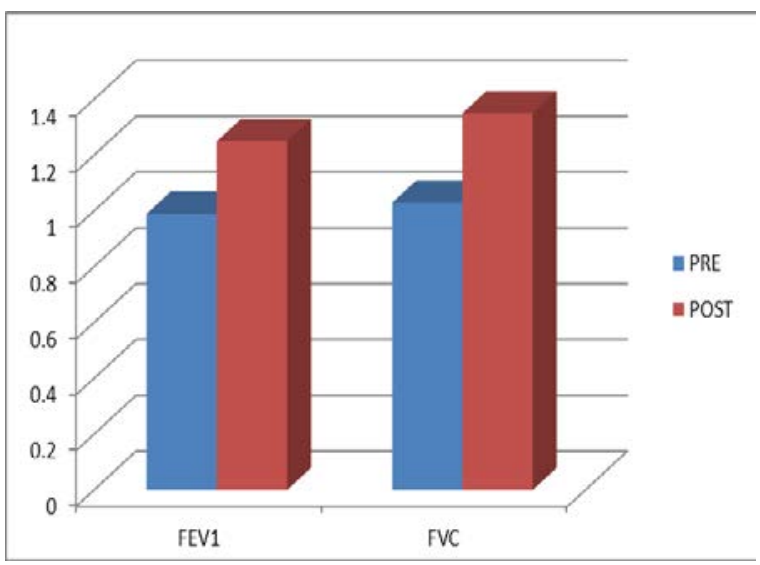

\section{Chest Expansion:}

Pre Intervention Mean of chest expansion was $1.15 \mathrm{~cm}$ and Post intervention Mean was $1.62 \mathrm{~cm}$ at Xiphoid Process Level

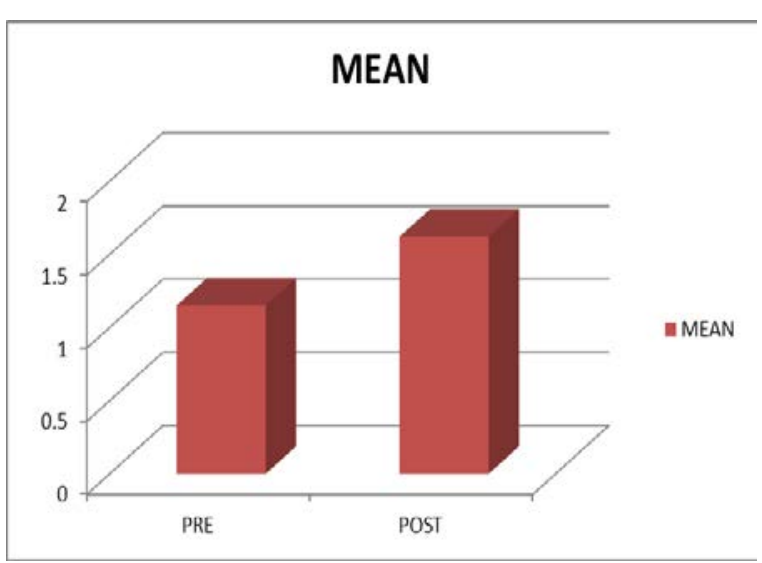



pulmonary functions in patients with spinal cord injury - a pilot study.

\section{DISCUSSION}

The present study "Effectiveness of Respiratory Proprioceptive Neuromuscular Facilitation Techniques on Pulmonary Functions in Patients with Spinal Cord Injury” was carried out at the dept. of Neuro Physiotherapy, Pravara rural hospital. In this study chest expansion was measured forced expiratory volume in one second, forced vital capacity, $\mathrm{FEV}_{1} / \mathrm{FVC}$ ratio and peak expiratory flow rate were taken as outcome measure to find out lung expansion and pulmonary ability of the participant respectively. The main purpose of this study was to see the effect of Respiratory PNF on Pulmonary Functions in Patients with Spinal Cord Injury. The result obtained in this study indicated that Respiratory PNF was more effective in improving Pulmonary Functions and chest expansion in Patients with Spinal Cord Injury.

Forced Expiratory Volume in one second $\left(F_{1}\right)$ and Forced Vital Capacity (FVC):

There was significant improvement of $\mathrm{FEV}_{1}$ and FVC after 4 weeks of intervention in patients with spinal cord injury the Pre Mean of FEV1 \& FVC 0.99 L \& 1.03L and Post Mean of FEV1 \& FVC was $1.25 \mathrm{~L} \& 1.35 \mathrm{~L}$ respectively. Where Chest Expansion was also improved in Respiratory PNF group (Pre Mean was 1.15 $\mathrm{cm}$ and Post Mean was $1.62 \mathrm{~cm}$ at Xiphoid Process Level) There were significant differences between both the groups at the end of the study. It means $\mathrm{FEV}_{1} / \mathrm{FVC}$ and chest expansion were more increased in Respiratory PNF group as compare to Conventional group.

Expiratory muscle function is more compromised than inspiratory muscle function among subjects with tetraplegia and high paraplegia, which can result in ineffective cough and propensity to mucus retention and atelectasis. Injury to the cervical and upper thoracic spinal cord disrupts function of inspiratory and expiratory muscles, as reflected by reduction in spirometric and lung volume parameters. ${ }^{[14]}$
Vikram M. and Kamaria K. (June 2012) conducted study on effect of intercostal stretch on pulmonary function Parameters and they stated that the use of manual stretching procedures has become more prevalent in cardiorespiratory physiotherapy to improve pulmonary functions. In the experimental group, subjects underwent intercostal stretch for ten breaths on the inspiratory phase of the respiratory cycle with breathing control exercises in semi recumbent position, while in the control group, breathing control exercises alone were performed in the semi recumbent position. The results of the study showed, $\mathrm{FEV}_{1}$ and FVC in the experimental group significantly improved than the control group, which means intercostal stretch increased lung volume and lead to improved lung function. This study suggested the intercostal stretching with breathing control may be more effective in improving dynamic lung parameters. ${ }^{[15]}$

The intercostal may enhance the chest wall elevation and thus increase expansion to improve intra-thoracic lung volume which contributes to improvement in flow rate percentage. This may contribute to the increase in ventilatory capacity (Chang et al., 2002).

The changes in ventilatory parameters may be due to the firing discharge of the muscle spindle during a passive stretch phase (Hirai et al., 1996). Intercostal stretching has activated the stretch receptors in the chest wall, thereby distending the thorax which could be neurologically linked to medulla with efferent nerve cells. It could also be argued that this altered ventilatory function may have resulted because of reflexive activation of the diaphragm by the intercostal afferents that innervate its margins (Jennifer and Pryor, 2008). Therefore, future design of stretching protocol in cardiorespiratory physiotherapy in neurological condition may be considered in order to promote ventilation. ${ }^{[15]}$ 
Komal Dattatray Thorat. Effectiveness of respiratory proprioceptive neuromuscular facilitation techniques on pulmonary functions in patients with spinal cord injury - a pilot study.

\section{CONCLUSION}

This Study concluded that Respiratory PNF increases Pulmonary Functions and chest expansion in Patients with Spinal Cord Injury after 4 weeks of Intervention.

\section{Acknowledgement: None}

\section{Conflict of Interest: None}

\section{Source of Funding: None}

\section{Ethical Approval: Approved}

\section{REFERENCES}

1. Kemal Nas, Levent Yazmalar, Volkan Şah, Abdulkadir Aydın, and Kadriye Öneș; Rehabilitation of spinal cord injuries; World J Orthop. 2015 Jan 18; 6(1): 8-16.

2. N Mathur, S Jain, N Kumar, A Srivastava, N Purohit \& A Patni: Spinal Cord Injury: Scenario in an Indian State; Spinal Cord 53, 349-352 (2015).

3. Gibson KL. Caring for a patient who lives with a spinal cord injury. Nursing. 2003; 33:36-41; quiz 42. [PubMed] [Google Scholar]

4. Fries JM. Critical rehabilitation of the patient with spinal cord injury. Crit Care Nurs Q. 2005;28:179-187. [PubMed] [Google Scholar]

5. Yildırım K, Şengel K. Spinal kord yaralanmaları ve rehabilitasyonu (Spinal cord injury and rehabilitation) Klnk Akt Tip Derg. 2004;(4):26-38. [Google Scholar]

6. William S. Linn, MA, Rodney H. Adkins, PhD, Henry Gong, Jr., MD, Robert L. Waters, MD Pulmonary Function in Chronic Spinal Cord Injury: A Cross-Sectional Survey of 222 Southern California Adult Outpatients; Arch Phys Med Rehabil 2000;81:757-63.

7. Schilero GJ, Spungen AM, Bauman WA, Radulovic $M$ and Lesser M (2009): Pulmonary function and spinal cord injury (Review). Respiratory Physiology and Neurobiology. 166(3): 129-41
8. Garstang SV, Miller-Smith SA (2007): Autonomic nervous system dysfunction after spinal cord injury. Physical Medicine Rehabilitation Clinics of North America 18: 275-96

9. Winslow C and Rozovsky J (2003): Effect of spinal cord injury on the respiratory system. American Journal of Physical Medicine and Rehabilitation 82(10): 8038141

10. Tollefsen E, Fondenes O (2012): Respiratory complications associated with spinal cord injury (Review). Tidsskrift for Den Norske Laegeforening. 132(9): 1111-4. (electronic, medline May 2013 - 22614314)

11. Consortium for Spinal Cord Medicine (2005): Respiratory management following spinal cord injury: a clinical practice guideline for health-care professionals. Washington, DC, Paralysed Veterans of America.

12. Wadsworth BM, Haines TP, Cornwell PL, Rodwell LT and Paratz JD (2012): Abdominal binder improves lung volumes and voice in people with tetraplegic spinal Cord injury. Archives of Physical Medicine and Rehabilitation 93(12): 2189-97

13. Park JH, Kang SW, Lee SC, Choi WA and Kim DH (2010): How respiratory muscle strength correlates with cough capacity in patients with respiratory muscle weakness. Yonsei Medical Journal 51(3): 392-7

14. Gregory J. Schilero,Marvin Lesser,Ann M. Spungen (2009): Pulmonary function and spinal cord injury.Respiratory Physiology and Neurobiology 166(3):129-141

15. Vikram m, badlisyah k, kamaria k., joseph L; effect of intercostal stretch on pulmonary function Parameters among healthy males; EXCLI Journal 2012;11:284-290

How to cite this article: Thorat KD. Effectiveness of respiratory proprioceptive neuromuscular facilitation techniques on pulmonary functions in patients with spinal cord injury - a pilot study. International Journal of Science \& Healthcare Research. 2021; 6(4): 186-190. DOI: https://doi.org/10.52403/ijshr. 20211026 